\title{
Utilization of Extracorporeal Membrane Oxygenation In Pediatric Cardiac Surgery: A Single Center Experience, 34 Cases in 8 Years
}

\author{
Cenap Zeybek, ${ }^{1}$ Mustafa Kemal Avsar,,3, ${ }^{2}$ Ozgur Yildirim, ${ }^{3}$ Arda Ozyuksel, ${ }^{3}$ and Mehmet Salih Bilal ${ }^{3}$ \\ ${ }^{1}$ Department of Pediatric Cardiology, Biruni University Faculty of Medicine, Istanbul, Turkey \\ ${ }^{2}$ Institute of Health Sciences, Istanbul Gelisim University, Istanbul, Turkey \\ ${ }^{3}$ Department of Cardiovascular Surgery, Medicana International Istanbul Hospital, Istanbul, Turkey \\ "Corresponding author: Dr. Mustafa Kemal Avsar, Medicana International Istanbul Hospital, Department of Cardiovascular Surgery, Beylikduzu Cad. No:3, Beylikduzu, \\ Istanbul, Turkey. Tel: +90-5324427701, E-mail: mustafakemalavsar@hotmail.com
}

Received 2017 June 12; Revised 2017 July 25; Accepted 2017 September 17.

\begin{abstract}
Background: Extracorporeal membrane oxygenation (ECMO) is used in a variety of indications worldwide. One of the most important subsets is postoperative congenital cardiac surgery cases unresponsive to conventional therapies. In this study, we present our ECMO experience in a single congenital cardiac surgery center.

Methods: ECMO was used in a total of 34 postoperative congenital cardiac surgery cases, whose operations had been performed by the same congenital cardiac surgery team. Patients' ages were between 3 days to 15 years. ECMO was used in four different indications; in case of unsuccessful weaning from cardiopulmonary bypass (OR-ECMO), in low cardiac output syndrome (LCOS-ECMO), in refractory post cardiac arrest (CPR-ECMO) and in respiratory insufficiency after RSV infection (RSV-ECMO).

Results: The follow-up period of patients ranged from 1 to 80 months, whereas ECMO duration ranged from 23 to 2218 hours. Six cases were OR-ECMO, 13 were LCOS-ECMO, 12 were CPR-ECMO and 3 were RSV-ECMO. Out of a total of 34 cases, 20 (58\%) cases were weaned from ECMO. Two of the patients, who were able to be weaned from ECMO passed away in the hospital; however, the other 18 patients (52.9\%) were discharged from the hospital without having any significant neurological deficits. The top survival rate (69\%) and weaning from ECMO was in the LCOS-ECMO group and the worst weaning from ECMO support (33\%) was in the RSV-ECMO. The worst survival rate (25\%) was in the CPR-ECMO group. Sepsis and associated multiple organ dysfunction were observed as the major cause of mortality in these patients. The most common complications were bleeding and mechanical complications related to cannulation.

Conclusions: ECMO may be required in postoperative congenital cardiac surgery cases in whom all other conventional therapies have failed. Indications, timing and maintenance of equipment are very important points in successful ECMO management. Increasing ECMO experience in the near future, will provide much decrease in mortality of congenital cardiac surgery.
\end{abstract}

Keywords: Extracorporeal Membrane Oxygenation, Pediatric Heart Surgery, Extracorporeal Life Support

\section{Background}

Extracorporeal membrane oxygenation (ECMO) is a lifesaving modality in postoperative cardiac surgery patients unresponsive to other therapies. Initially starting from 1970s, the usage of ECMO in pediatric cardiac surgery has gradually increased over the last 40 years $(1$, 2 ). The most common indications for ECMO usage in postoperative pediatric cardiac cases are low cardiac output syndrome, pulmonary hypertension, inotropic refractory cardiogenic shock, inability to weaning from cardiopulmonary bypass, bridging to cardiac transplantation and extracorporeal cardiopulmonary resuscitation.

The survival chance of children with ECMO treatment after cardiac surgery, although varying according to different centers, has been reported to be between 38 and 55\% (3-9). The causes of the high mortality rate are multifactorial. However, the primary reasons are ECMO-related com- plications, errors in ECMO management, delay of the decision to implement ECMO or decannulation, complex congenital cardiac pathologies and post-operative significant residual defects (10).

In this study, we would like to present our ECMO experience including management strategies, complications, and survival rates accompanied by the literature as implemented in 34 pediatric patients.

\section{Methods}

This study includes 34 patients who have undergone ECMO support after cardiac surgery between 2009 and 2016. Following the approval of the institutional review board, the medical data of these patients were evaluated retrospectively. During this period, a total of 1,620 pediatric cardiac surgeries had been performed and ECMO 
was deemed mandatory in $2 \%$ of these cases. Apart from one case, ECMO support was not applied to any of the other cases before the surgery. ECMO support was implemented in 4 different indications including: (i) ECMO application in cases of failing to wean from the cardiac operation with cardiopulmonary bypass (Operation Room ECMO; OR-ECMO), (ii) ECMO application in postoperative low cardiac output syndrome (Low cardiac output syndrome ECMO; LCOS-ECMO), (iii) after postoperative refractory cardiac arrest (Cardiopulmonary resuscitation ECMO; CPR-ECMO) and (iv) ECMO application in the case of respiratory insufficiency after respiratory syncytial virus infection (Respiratory Syncytial Virus ECMO; RSV-ECMO).

The ages of 13 female and 21 male patients ranged between 3 days and 15 years. Veno-venous ECMO was applied to one of the cases whereas veno-arterial ECMO was applied in the rest. In one of the cases, wherein an emergency double switch operation was performed, in the second day of operation, the oxygenator has been removed by changing the mechanical circulatory support to a leftto-left system. Within the exemption of one case, cannulation was performed in ascending aorta and right atrium for arterial and venous lines, respectively. In one case, we initiated the ECMO support with femoral artery and vein cannulation. ECMO support was provided with a roller (Stockert S3: Sorin Group Deutschland GmbH Lindberghstr. Munich, Germany) and/or centrifugal (Stockert SCP; Sorin Group Deutschland GmbH Lindberghstr. Munich, Germany) pump. A Dideco (Sorin Group Italia, Mirandola, Italy) pediatric oxygenator and heparin-coated lines were used in the ECMO setup.

In all patients, ECMO circuits were primed with fresh whole blood and crystalloid solutions were not used. According to the patient's coagulation status, 2 -3 minutes before the cannulation, heparin was administered at a dose of $30-50 \mathrm{IU} / \mathrm{kg}$. Pump flow was set at $120-160 \mathrm{~mL} / \mathrm{kg} / \mathrm{min}$. Mean arterial pressure was kept around $40 \mathrm{mmHg}$ in newborns, $50 \mathrm{mmHg}$ in children and $60 \mathrm{mmHg}$ in adolescents. When ECMO was initiated in patients with a surgical history of a central shunt or Blalock Taussig shunt, the shunt was clamped. After ECMO initiation, the ventilator settings were changed to rehabilitate the lungs. The ventilator parameters were set as positive end-expiratory pressure (PEEP) of 8 to $10 \mathrm{~cm} \mathrm{H}_{2} \mathrm{O}$, inspired oxygen fraction $\left(\mathrm{FiO}_{2}\right)$ of 0.3 , tidal volume of 3 to $5 \mathrm{ml} / \mathrm{kg}$, peak inspiratory pressure of $25 \mathrm{~cm} \mathrm{H}_{2} \mathrm{O}$ or less and ventilation rate of 10 to 15 breaths/min. While the temperature of the system was adjusted to normal body temperature in patients without cardiac arrest, in the cardiac arrest patients, the patients were cooled down to $33-35^{\circ} \mathrm{C}$ in the first 24 hours. The sternum of the patients that underwent central ECMO was left open and their skin was closed with polytetrafluoroethy- lene patch. After the evaluation of neurological status of patients, infusions of muscle relaxants, sedatives (midazolam) and analgesics (fentanyl) were initiated. In only one patient, who underwent peripheral ECMO, extubation was performed following the first 24 hours of ECMO implementation. Gram positive and gram negative broadspectrum antibiotherapy was administered to all of the patients. After ECMO support was started, vasopressor and inotropic agents were terminated; patient's liquid balance were tried to be kept in negative, and when necessary diuretics were administered. Accordingly, modified ultrafiltration was implemented to patients who did not respond to diuretics satisfactorily. Modified ultrafiltration target was scheduled to hold a negative balance of $10-20 \mathrm{~mL}$ per hour. All vital parameters were monitored; transcranial ultrasound was performed in patients when necessary.

Hourly arterial blood gas analyses were performed till the patients' situation was stable. Activated clotting time (ACT) was evaluated quarterly. A complete blood count and blood biochemistry were evaluated daily. Invasive monitorization catheters were replaced when necessary; blood and other cultures were taken weekly. Daily chest $\mathrm{X}$-ray was taken; cerebral or thoracic computed tomography with ECMO transport was performed to patients if needed. The ACT values were kept between 180 - 200 seconds with continuous heparin perfusion, which was measured with Hemacro Jr. Signature Elite (International Technidyne Corporation, Edison, NJ, USA) device. The heparin perfusion was paused during severe mediastinal, intracranial or gastrointestinal hemorrhage. Oxygenators and tubing sets were urgently changed in the presence of clot. The oxygenator was also immediately replaced in case of deterioration in the patient's oxygenation and occurrence of hematuria. Hematocrit and thrombocyte counts were kept over 30 and $100,000 / \mathrm{mm}^{3}$, respectively with blood and thrombocyte transfusions when necessary.

One of the most important points that we observed during ECMO implementation was the decision for the termination of ECMO and decannulation. For this decision making, cardiac and pulmonary parameters were carefully evaluated along with observation of the general conditions of the patient. Cardiac evaluation was evaluated by transthoracic echocardiography. Echocardiography was performed at least once per day during the ECMO process during which the ECMO flow was temporarily reduced to $30 \%$ of normal. The cardiac parameters such as ventricular functions, left ventricular and left atrial dimensions, valvular regurgitation, any residual intracardiac defects/shunts and pulmonary artery pressure were evaluated. For the pulmonary assessment, we evaluated the radiological appearance of the parenchyma, ventilation parameters and tidal volume. Minute doses of inotropic support (0.03 mi- 
crograms $/ \mathrm{kg} / \mathrm{min}$ epinephrine and $0.5 \mathrm{mg} / \mathrm{kg} / \mathrm{min}$ milrinone) were started in the period of the ECMO weaning. The criteria for weaning from ECMO were determined as cardiac functions returning to normal in echocardiography, normal values of blood gas and laboratory data, lactate level below $2 \mathrm{mmol} / \mathrm{L}$, mixed venous oxygen saturation over 65\%, normalization of chest radiographs and ventilator parameters. While weaning from ECMO, the flow was reduced by 50-100 ml per minute flow rate and ECMO was terminated in a maximum of 12 hours. The recirculation flow was started after the bridging connection between arterial and venous systems and after placing the clamp to the cannulas. In case of unexpected hemodynamic deterioration, increased lactate levels and acidosis, the mechanical circulatory support was started again. While patients on ECMO were isolated from other patients, nursing care was provided by one person. After evaluation of hemodynamic parameters and blood gas analysis as well as echocardiographic studies, decannulation was performed in the following 6 hours of termination of the ECMO. The sternum of the patients was closed in the following day.

\section{Results}

Extracorporeal membrane oxygenation support was applied in $34(2.09 \%)$ cases out of a total of 1620 pediatric heart surgery patients who were operated in our center in the last eight years by the same surgical team. The follow-up period of patients was ranging between 1 and 80 months. ECMO duration ranged from 23 to 2218 hours. Six patients were OR-ECMO, 13 patients were LCOS-ECMO, 12 patients were CPR-ECMO and 3 patients were RSV-ECMO. The demographic characteristics of the patients, diagnosis, operative data, ECMO weaning state, neurological deficits, mortality, causes of mortality and duration of ECMO are demonstrated in Table 1.

Out of a total 34 cases with ECMO support, 20 cases (58\%) were successfully weaned. Two of the patients, who were able to be weaned from ECMO passed away in the hospital; however, the other 18 patients (52.9\%) were discharged from the hospital without having any significant neurological deficits. The top survival rate was in the LCOS-ECMO group (69\%), whereas the worst rate of weaning from ECMO support was in the RSV-ECMO population (33\%). In addition, the worst survival rate (33\%) was in the CPR-ECMO group. Sepsis and associated multiple organ dysfunctions were observed as a major cause of mortality in these patients. The most common complications were bleeding and mechanical complications related to ECMO. For simplicity, the results will be discussed under 4 sep- arate subgroups as LCOS-ECMO, OR-ECMO, CPR-ECMO and RSV-ECMO.

- Group 1 (OR-ECMO): 6 of 34 (17.6\%) cases in our patient population were OR-ECMO. It was performed in the case of not being able to wean the patient from cardiopulmonary bypass. In 4 patients, ECMO was successfully completed (66.6\%) whereas 2 patients passed away. The cause of mortality in one patient was multi-organ dysfunction syndrome (MODS) due to sepsis. The second patient was lost due to accidental displacement of the aortic cannula during sternum undressing and transport. Among the surviving patients, there was no evidence of any neurological complications. We performed mediastinal revision for bleeding in 3 of the cases in the intensive care unit. Late mortality was not encountered in this group.

- Group 2 (LCOS-ECMO): the indication in 13 patients out of 34 cases (38.2\%) was ECMO implementation due to low cardiac output status. In this group, 10 out of 13 patients (76.9\%) were weaned from ECMO. 9 cases (69\%) that were weaned successfully from ECMO support were discharged from the hospital. After ECMO implementation, mortality was observed in one case related to significant cerebral infarction. Along with this, the cause of mortality in two patients was MODS due to sepsis. The cause of mortality encountered in another patient was exsanguination following an unexpected rupture of the lines. In 9 discharged patients in this group, two had cerebral infarction with insignificant neurological sequelae. The remaining 7 patients were in good neurological condition. A repeat ECMO implementation was deemed necessary between the termination of the circulatory support and decannulation periods due to hemodynamic and blood gas disturbances in two patients in this group, both of whom were weaned successfully at the end. In one of these patients, a bidirectional cavopulmonary anastomosis and a concomitant pulmonary artery banding was performed. This patient failed for 3 times while weaning from the ECMO support. This patient was reoperated under ECMO support and a Hemi-Mustard operation was performed. Late mortality in LCOS-ECMO group was not observed. Two of patients in this group underwent a revision due to mediastinal bleeding.

- Group 3 (CPR-ECMO): 12 out of 34 ECMO patients (35.2\%) needed mechanical circulatory support following cardiopulmonary arrest. The unexpected cardiopulmonary arrest incidence was susceptible (12 arrests in 1620 patients, $<1 \%$ ). In this group of patients except for a single case, the CPR-ECMO was implemented in intensive care unit when there was no response to medical and mechanical cardiopulmonary resuscitation at the 20th minute of external cardiac massage. The exceptional case was a 15year-old male with the diagnosis of ventricular septal defect and pulmonary atresia. This patient had undergone 
Table 1. Demographic Characteristics of the Patients

\begin{tabular}{|c|c|c|c|c|c|c|c|c|}
\hline Age & Diagnosis & Type of Operation & Duration, $h$ & Wean & Mrt & oxy\# & ECMO Group & UF \\
\hline $21 \mathrm{mo}$ & Multiple VSD + PH & Multiple VSD Closure & 2218 & Yes & None & 4 & 2 & Yes \\
\hline $13 \mathrm{yr}$ & s/p Fontan + AV Regurgitation & Fenestration Closure, AV Valve Plasty & 1416 & No & Yes & 5 & 3 & Yes \\
\hline $6 \mathrm{mo}$ & TOF, Hypoplastic PA & Left MBTS & 1176 & No & Yes & 3 & 3 & Yes \\
\hline $22 \mathrm{~d}$ & Truncus Arteriosus Type I & Total Correction & 1116 & No & Yes & 2 & 1 & Yes \\
\hline $7 \mathrm{mo}$ & TAPVR & TAPVR Correction & 868 & No & Yes & 3 & 2 & Yes \\
\hline $3 \mathbf{m o}$ & AVSD (Rastelli Type A) & Total Correction & 816 & No & Yes & 2 & 1 & Yes \\
\hline $9 \mathrm{mo}$ & TOF, PA, PDA & Central Shunt, PDA Ligation & 745 & No & Yes & 2 & 2 & Yes \\
\hline $8 \mathrm{mo}$ & VSD, PS, TA & Bilateral Bidirectional Glenn procedure & 744 & No & Yes & 2 & 3 & Yes \\
\hline $8 \mathrm{mo}$ & TOF, PA & Central Shunt & 650 & No & Yes & 3 & 3 & Yes \\
\hline $1 \mathrm{yr}$ & AVSD (Rastelli Type A) & Total Correction & 650 & Yes & Yes & 3 & 2 & Yes \\
\hline $1 \mathrm{yr}$ & TOF & Total Correction & 410 & Yes & None & 2 & 1 & None \\
\hline $2 \mathrm{yr}$ & TOF & Total Correction & 408 & Yes & None & 1 & 3 & None \\
\hline $4 \mathrm{mo}$ & TGA & Arterial switch operation & 363 & Yes & None & 2 & 3 & None \\
\hline $9 \mathrm{yr}$ & s/p Glenn, DORV, PS & Fontan, $\mathrm{AV}$ valve plasty, atrial septectomy & 330 & No & Yes & 2 & 3 & Yes \\
\hline $2 \mathrm{yr}$ & $s / p V S D+P A B$ & Pulmonary Debanding, pulmonary patchplasty & 288 & Yes & None & 1 & 2 & Yes \\
\hline $3 \mathbf{y r}$ & DORV,PS,VSD, s/p Brock Operation & Total Correction, Transannular Patch & 264 & No & Yes & 2 & 3 & Yes \\
\hline $7 \mathrm{mo}$ & TGA+ASD & Hemi-Mustard operation & 262 & Yes & None & 3 & 2 & None \\
\hline $1 \mathrm{yr}$ & Unbalanced AVSD, DORV, PS & Bidirectional Glenn procedure & 254 & No & Yes & 1 & 4 & None \\
\hline $10 \mathrm{mo}$ & TGA & Arterial switch operation & 236 & Yes & None & 1 & 2 & None \\
\hline $6 \mathrm{mo}$ & TOF, PA & Right MBTS & 216 & No & Yes & 2 & 3 & None \\
\hline $5 \mathrm{mo}$ & AVSD (Rastelli Type A) & Total Correction & 208 & Yes & None & 2 & 2 & None \\
\hline $8 \mathrm{mo}$ & $\mathrm{ASD}+\mathrm{PH}$ & ASD closure & 206 & Yes & None & 1 & 2 & None \\
\hline $9 \mathrm{mo}$ & $c-T G A+s / p$ PAB & DSO & 196 & Yes & None & 1 & 1 & None \\
\hline $18 \mathrm{yr}$ & Cardiac arrest, s/p VSD, PS & V-A ECMO & 144 & Yes & None & 1 & 3 & Yes \\
\hline $8 \mathrm{mo}$ & DORV, VSD, PS & Atrial septectomy, PAB, BDG & 92 & Yes & None & 1 & 4 & None \\
\hline $4 \mathrm{mo}$ & $s / p$ Central shunt + TAPVR & Total Correction & 88 & Yes & None & 1 & 2 & None \\
\hline $8 \mathrm{mo}$ & $\mathrm{IAA}+\mathrm{PDA}+\mathrm{PH}+\mathrm{DILV}+\mathrm{MVA}$ & IAA Repair, atrial septectomy, PDA ligation, PAB & 81 & Yes & None & 1 & 2 & None \\
\hline $10 \mathrm{mo}$ & TOF & Total correction & 79 & Yes & None & 1 & 2 & None \\
\hline $9 \mathrm{mo}$ & VSD & VSD Closure & 75 & No & Yes & 1 & 4 & Yes \\
\hline $11 \mathrm{mo}$ & TOF & Total correction & 72 & No & Yes & 1 & 3 & None \\
\hline $8 \mathrm{mo}$ & Ebstein's anomaly $+\mathrm{ASD}+\mathrm{TR}$ & Tricuspid plasty + ASD Closure & 70 & Yes & None & 2 & 1 & Yes \\
\hline 3d & HLHS & Norwood Stage I & 60 & No & Yes & 1 & 2 & Yes \\
\hline $10 \mathrm{mo}$ & TGA, VSD, ASD & Senning + VSD closure & 37 & Yes & Yes & 1 & 3 & None \\
\hline $7 \mathrm{mo}$ & ASD, VSD, PDA, PH & AV Valve Plasty + PDA Ligation + PAB & 23 & Yes & None & 1 & 1 & None \\
\hline
\end{tabular}

Abbreviations: ASD, atrial septal defect; AV, atrioventricular; AVSD, atrioventricular septal defect; c-TGA, congenitally corrected transposition of the great arteries; DILV, double inlet left ventricle; DORV, double outlet right ventricle; DSO, double switch operation; HLHS, hypoplastic left heart syndrome; IAA, interrupted aortic arcus; MBTS, modified Blalock-Taussig Shunt; mo, months; Mrt, mortality; MVA, mitral valve atresia; Oxy\#, number of oxygenator replacement; PA, pulmonary atresia; PAB, pulmonary artery banding; PDA, patent ductus arteriosus; PH, pulmonary hypertension; PS, pulmonary stenosis; TA, tricuspid atresia; TAPVR, Total abnormal pulmonary venous return; TGA: transposition of the great arteries; TOF, tetralogy of Fallot; TR, tricuspid regurgitation; VA, veno-arterial; VSD, ventricular septal defect; UF: ultrafiltration; yrs, years.

a Rastelli operation with a $19 \mathrm{~mm}$ pulmonary heterograft years ago, and 6 months before the event of cardiac arrest, an injectable pulmonary valve was implanted via femoral vein by the interventional cardiology. He was admitted to our emergency department with severe respiratory distress and cardiac arrest occurred there. The spontaneous cardiac beats were detected at the 10th minute of resuscitation whereby he was taken into cardiac surgery intensive care unit. Femoral veno arterial ECMO was performed under maximal inotropic support because the patient had hypotension, bradycardia and significant arrhythmia. The patient waked up after 24 hours and ECMO support was continued while the patient was awake. In this group, the weaning from ECMO was achieved in 5 (41.6\%) cases. Four patients out of 5 were discharged, whereas in one case, late mortality occurred in the hospital due to severe cerebral infarction. Along with this case, in CPR-ECMO group, mortality was observed in 8 (66.6\%) patients. The following reasons were identified as a cause of mortality: MODS due to sepsis in 4 cases, disseminated intravascular coagulation (DIC) in 2 cases and cerebral infarction in 2 cases. No lateterm mortality was observed among discharged patients in this group.

- Group 4 (RSV-ECMO): An ECMO support was imple- 
mented in 3 of our patients with RSV infection. One (33.3\%) of the patients was weaned from ECMO and was discharged without having any problem. Veno-venous and veno-arterial ECMO was implemented in two cases and one case, respectively. The causes of mortality observed in these two cases were sepsis and hypoxic encephalopathy, respectively.

The overall causes of mortality observed in our patient population are demonstrated in Table 2.

Table 2. Causes of Mortality

\begin{tabular}{lc}
\hline Causes of mortality & No. (\%) \\
\hline Sepsis + MODS & $9(57)$ \\
\hline Massive consumption coagulopathy & $2(12)$ \\
Hypoxic-ischemic brain injury & $3(19)$ \\
Mechanical complications & $2(12)$ \\
Total & 16 \\
\hline
\end{tabular}

Abbreviation: MODS, multiple organ dysfunction syndrome.

\section{Discussion}

The indications for ECMO implementation are variable among many health centers over the world. When cardiac surgery is concerned, failure of weaning from CPB after the operation, postcardiotomy cardiac and pulmonary failure and cardiopulmonary resuscitation for refractory cardiac arrest may be focused on. Despite the technological developments and increased experiences, the chances of survival after cardiac surgery with ECMO implementation in children remain between 38\% - 55\% (3-9).

According to study carried out by Chrysostomou and colleagues in a patient population of 3,524 children who underwent congenital heart surgery, 95 (3\%) required ECMO and their short-term and mid-term survival rate were $73 \%$ and $66 \%$, respectively (10). In our patient population, 34 patients out of a total of 1,620 congenital cardiac surgery cases, 34 (2\%) of them required ECMO support. Twenty (58\%) of these patients were weaned from ECMO and 18 (52.9\%) were discharged. Although Chrysostomou and colleagues stated that the chance of survival in CPRECMO group was as high as 75\%, some studies report worse results ranging between $30 \%$ and $50 \%(5,6,11,12)$. In our study, the lowest chance of survival was observed in the CPR-ECMO and RSV-ECMO groups. Furthermore, Chrysostom et al reported that the chance of survival in OR-ECMO and LCOS-ECMO groups were as high as $77 \%$ and $62 \%$, respectively. In our study, the highest rate of survivors was in the LCOS-ECMO group with $69 \%$ and this was followed by the OR-ECMO group with a survival rate of $66 \%$. The lowest survival chances were encountered in the CPR-ECMO and the RSV-ECMO groups in our patient population with a rate of 33\%. The extracorporeal life support organization (ELSO) Registry report states that in CPR-ECMO the chance of survival rate was $39 \%$ in neonates and $40 \%$ in children; and in cardiac etiology for the implementation of ECMO, the chance of survival was $40 \%$ in neonatal and $49 \%$ in pediatric patients (13).

According to a large multicenter study, the prolonged period of ECMO reduces the chance of survival and there is no significance difference in mortality below 7 days of duration of ECMO. However, after 7 days and beyond, every additional 24 -hour increased the mortality by $18 \% 2$. In our study, we found that in cases where ECMO was applied for less than 7 days, the mortality rate was 30\% (3 of 10 patients); whereas in cases where ECMO support was applied for seven or more days, mortality range was $60.8 \%$ (14 of 23 patients). Only one out of 10 patients whom we supported more than 410 hours (about 17 days) with ECMO support survived. We observed 65\% survival rate (15 of 23 patients survived) in the patient population who were treated with ECMO support for 410 hours or less. Ultimately, we think that with prolonged ECMO duration, several factors such as ECMO associated bleeding, infection, increases of complications as central nervous system complications, increased mechanical ventilation support, observation of multiple organ failure, and increased length of stay in intensive care unit are responsible for increased mortality rate. Gupta et al. in their study related to children with acute respiratory and cardiac failure reports that in cases that were treated with ECMO duration within 28 days and above the incidence of 4 and more organ dysfunctions was encountered in 69\%. Moreover, they observed neurological deficits in more than half of their patients, thromboembolic and hemorrhagic complications were observed as $25 \%$ and $37 \%$, respectively (14). According to study conducted by Hintz et al. (15) and Aharon et al. (16), the critical duration for decent survival of children who underwent cardiac surgery was provision of 3 days or below ECMO support.

In ELSO reports, ECMO complications were classified into two groups: complications associated with patient such as intracranial hemorrhage, cannula site bleeding, surgical site bleeding, cardiac tamponade, clinical seizures and mechanical complications such as cannula problems, tube rupture, pump malfunction, oxygenator failure (13). In our patient population, we encountered mechanical complications in almost all of the patients who were treated with ECMO support over 10 days. One of the patients passed away at the 1176th hour of ECMO treatment during the dressing due to accidental displacement of the 
aortic cannula. Another annoying mechanical complication occurred in 745th hour of ECMO treatment at the head of the roller pump due to rupture of tubing set and this patient also passed away. The position of cannula should be checked when the echocardiography is performed. It is very important to place aortic and venous cannulas properly in the right place. Placement of the aortic cannula very close to the valve may result in aortic regurgitation and cardiac perfusion defect, whereas placement of aortic cannula very distal, may disturb the cerebral perfusion. Therefore, in the aortic cannulation for ECMO treatment, we choose the DLP brand (Medtronic Inc., Minneapolis, MN, USA) wire guided aortic cannula. The diameter of the cannula is selected according to weight of the patient and they were placed approximately 1.5 to $2 \mathrm{~cm}$ distal to the aortic root. It is very important to ensure adequate return with appropriate venous cannulation on ECMO. Venous cannulation from the right atrium was achieved with the metaltipped venous cannula. In our cases, we did not perform left atrial cannulation for left ventricular decompression. In some centers, the left ventricular decompression is performed routinely during ECMO and the advocates of this opinion stated that left ventricular decompression is a significant point in the success of ECMO15, (17).

Oxygenator failure was observed in almost all of our cases lasting longer than 10 days. During the ECMO treatment, as a part of the protocol, ECMO oxygenator was changed in every 10-14 days. However, in the presence of oxygenation problems, hemolysis and hematuria in patients, we changed oxygenator regardless of time. In patients, whereby heparin treatment was suspended due to bleeding in the presence of thrombus in the cannula or in tubing system, or under heparin treatment in tubing system or in the cases of thrombus detected in cannulas, we changed cannula(s) and/or tubing sets without waiting for the occurrence of any complication. We observed that as the team's experience increased, the replacement of the tubing system decreased from 10 minutes to 1 minute. During the replacement process of cannula, the second cannulas were positioned firstly by placing a second purse string in aorta or to the right atrium. Subsequently the previous cannulas were removed, purse stitches were tied, bleeding control was achieved and new cannulas were mounted on the ECMO system. We believe that these problems are inversely proportional with surgical team's and perfusionist's experience. In our congenital cardiac surgery center, the head of the surgical team and chief perfusionist were the same people throughout the stated time interval. A significant amount of 'know how' experience is accumulated in our clinic with more than 4.000 cases in the last decade. A pre-setup algorhythm and management protocols are produced in order to systematically manage any patient with any complication from admission to the hospital until discharge.

One of the most serious complications of ECMO related to patient is bleeding. In case of bleeding, heparin infusion may be ceased for a while. It has been reported that ECMO implementation without heparin infusion is feasible between 1 - 28 hours when the heparin coated circuits are used (18). In case of a bleeding situation, we preferred intermittent heparin administration rather than continuous infusion or complete cessation of heparin infusion. However, in one of our cases with severe cerebral hemorrhage, we had to stop heparin administration for one week out of 92 days of support and we continued ECMO support with heparin-coated tubing. During this period, the lines and the oxygenator were replaced for two times and the patient was successfully discharged with minor seizures. Bleeding can be seen in any organ systems or at the cannulation sites, as well. If necessary, mediastinum can be opened and bleeding control can be performed in intensive care unit. Furthermore, in case of bleeding in other organs such as brain or gastrointestinal system, we believe that heparin should be discontinued and it should not be given until acute hemorrhagic period ends. It has been reported that in cases of discontinued heparin, application of nitric oxide and prostacyclin into the system may prevent platelet activation, adhesion, and aggregation until the bleeding due to heparin is controlled (19). There are also ongoing studies related to ECMO treatment without heparin as well (20).

Hervey and colleagues reported that three most common neurological complications related to ECMO implementation are cerebral hemorrhage, central nervous system infarcts and seizures (21). Cerebral hemorrhage is the most common complications of ECMO application and in early life and cerebral complications decrease when the age increases (13). Cerebral hemorrhage is termed as a malignant complication, since it is often the primary cause of mortality (22). In order to avoid these complications, routine use of heparin coated sets and a better selection of anticoagulation management regimen (thromboelastography, platelet aggregation tests) may be suggested. In our study, cerebral hemorrhage was observed in 3 patients and in 2 of them mortality occurred, whereas one patient was discharged.

Many studies have shown that the application of ECMO may cause deterioration in renal functions and acute renal injuries that can lead to increase in mortality rates (2325). Nephrotoxic agents used in the patients treated with ECMO lead to decreased renal perfusion during ECMO support and infections, blood transfusions, systemic inflammatory reaction syndrome and hemolysis may be associated with renal damage. In 15 out of our 34 cases, acute re- 
nal failure occurred in the first three days. In the study conducted by Zwiers and colleagues, it was observed that in a total of 242 cases, $63 \%$ of patients developed acute renal injury, and in most of these cases acute renal injury was observed in the first 2 days of ECMO treatment (26). The treatment of acute renal failure is managed with fluid restriction, diuretic therapy, peritoneal dialysis, hemodialysis in ECMO system and renal replacement therapy including hemodiafiltration. Higher mortality rates have been observed in cases of ECMO with acute renal damage requiring renal replacement therapy (27). In 10 out of our 14 cases, liquid administration modifications and normal urine output was ensured and the renal damage was treated with medication alone. However, in 4 cases, modified hemodiafiltration was deemed mandatory. We implemented peritoneal dialysis in one case and 3 of these cases died due to sepsis. The other causes of mortality in our patient population were hypoxic-ischemic brain injury 3 cases, massive consumption coagulopathy in 2 cases, in mechanical complications of ECMO in one case and accidental removal of the arterial cannula in another case. The mechanical complication was due to the explosion of roller pump head.

Infections (sepsis), cerebral hemorrhage and renal insufficiencies are major causes of death in patients treated with ECMO (28). In our study, the most common cause of mortality seen in 16 patients was sepsis with a rate of $56 \%$ and related multiorgan failure (with involvement of at least 3 organ systems) that was encountered in 9 of 16 cases. However, the age, diagnosis and the duration of ECMO are not similar in our subgroups, which obviously affected the morbidity and mortality results. When the ECMO duration gets longer, susceptibility to infection increases due to presence of invasive catheters, the open sternum with a PTFE patch, factors related to health personnel in the intensive care unit, daily dressings, bleeding or other problems. As soon as we start with the ECMO treatment, broad spectrum antibiotics with gram-positive and gram-negative effect are administered to all of our patients. We take catheter tip cultures with daily wound site culture, deep tracheal aspiration culture, and blood cultures when there's a febrile attack. Antibiotherapy is then set up according to the results of the antibiograms. In cases with a prolonged antibiotic therapy for longer than 2 weeks we generally add antifungal agents, as well. The patient follow-up during the ECMO period is provided by a single nurse and support personnel, in order to reduce contact with other patients. Intravenous lines such as arterial, central venous catheters and urinary catheter are replaced every 10 days for reducing the risk of catheter infection. The patient's chest dressings are completely implemented in a sterile environment.

The indication for ECMO implementation following congenital cardiac surgery may be various and in our study the most common pathology requiring ECMO support was Tetralogy of Fallot (TOF). Similarly, in the study of Balasubramanian and colleagues, the most common pathology was TOF with an incidence of $30 \%$ in 53 cases (29). In another study, the most common pathology for ECMO implementation was reported to be arterial switch operation (30). Although we did not reveal a significant difference in our patient population, ECMO implementation in patients with two-ventricle (biventricular) repair is reported to be more successful than ECMO treatment in cases following with univentricular palliation (16). On the other hand, Morris and colleagues did not report such a difference in their patient series (3).

One of the main points for a successful recovery following the ECMO support is the presence of a complete cardiac surgery meaning that the heart has a good biventricular function without any residual defects or shunts following the operation. In cases of ECMO treatment during incomplete repair of hemodynamically significant residual defects, the mortality rates are reported to be as high as $100 \%$ (31). A completion surgery is deemed mandatory in such cases. We did not encounter such a problem in our study.

There are large number of studies and conflicting results in ECMO treatment regarding indicators of mortality. A variety of factors are used to identify poor prognosis in ECMO practice such as age, sex, cardiac physiology (univentricular or biventricular), type of repair, prolonged CPB and cross-clamp times, use of total circulatory arrest, utilization of intraoperative ultrafiltration, anesthetic agents, renal failure, hepatic failure, mediastinal hemorrhage, cerebral hemorrhage, cardiac arrest time, infections, reasons related with pump, end-organ damage that may occur during ECMO (3, 31-33). Low $\mathrm{pH}$ values are the only factor representing poor hospital survival during ECMO treatment and it was stated that at the lowest $\mathrm{pH}$ levels, in 48 hours when the highest level of lactate is reached, death is almost inevitable (33). Duration of cardiopulmonary resuscitation until the implementation of ECMO in patients with cardiac arrest has not been clarified yet. However, according to our opinion, initiating ECMO support faster and shortening the CPR duration may increase the chance of survival and reduce cerebral complications. In the study conducted by Walter and colleagues wherein ECMO support was performed in 42 children after cardiac arrest, it was found that the average CPR duration in survived 17 children was 30 minutes, whereas in 25 patients, where mortality had been observed, the average CPR duration was 46 minutes (34). It was pointed out that extension of CPR duration and usage of high-dose inotropic treatment was associated with increased mortality 
(34). Moreover, CPR with a chest compression performed accurately can merely provide $20 \%-30 \%$ of the systolic cardiac output (35). Chen et al. pointed out that if the starting time of ECMO support is under 60 minutes in cardiac arrest, the survival rate of patients is higher (36). Therefore keeping a non-primed but ready ECMO system in pediatric cardiac surgery centers would obviously aid in reducing the initiation duration of ECMO. We keep centrifugal ECMO system ready for emergencies in the intensive care unit. Awake ECMO can also be a choice of treatment modality where appropriate peripheral ECMO implementation strategy is available. Extubation of these patients will eliminate the negative effects of ventilatory support. We have performed this in one of our patients and achieved an encouraging result.

As a result, the implementation of ECMO has now become an integral part of pediatric cardiac surgery. In our study, ECMO was performed with $52 \%$ survival success and with concomitant mild neurological deficits in patients who underwent cardiac surgery with the diagnosis of congenital heart defects in our center. Our success momentum is developing with a strict ECMO protocol in our clinic, training of the surgeons, perfusionists, nurses and health personnel. We believe that the survival rate will increase with the improvement of practices in clinics utilizing ECMO.

\subsection{Limitations}

Our primary aim was to present and discuss our clinical experience with ECMO implementation in different subgroups undergoing congenital cardiac surgery. Our data is composed of retrospective analysis, therefore an important limitation is the non-similar demographic properties of the subgroups. Moreover, age of the patients, diagnosis, as well as the duration of the ECMO will obviously affect the morbidity and mortality rates when these groups are compared. Secondly, the ages, diagnosis and the duration of ECMO of our patients were not similar in the subgroups, which obviously affected the morbidity and mortality results.

\section{References}

1. Soeter JR, Mamiya RT, Sprague AY, McNamara JJ. Prolonged extracorporeal oxygenation for cardiorespiratory failure after tetralogy correction. J Thorac Cardiovasc Surg. 1973;66(2):214-8. [PubMed: 4578586].

2. Gupta P, Robertson MJ, Beam B, Gossett JM, Schmitz ML, Carroll CL, et al. Relationship of ECMO duration with outcomes after pediatric cardiac surgery: a multi-institutional analysis. Minerva Anestesiol. 2015;81(6):619-27. [PubMed: 25280142].

3. Morris MC, Ittenbach RF, Godinez RI, Portnoy JD, Tabbutt S, Hanna BD, et al. Risk factors for mortality in 137 pediatric cardiac intensive care unit patients managed with extracorporeal membrane oxygenation. Crit Care Med. 2004;32(4):1061-9. [PubMed: 15071402].
4. Alsoufi B, Al-Radi OO, Gruenwald C, Lean L, Williams WG, McCrindle BW, et al. Extra-corporeal life support following cardiac surgery in children: analysis of risk factors and survival in a single institution. Eur J Cardiothorac Surg. 2009;35(6):1004-11. doi: 10.1016/j.ejcts.2009.02.015. [PubMed: 19356943] discussion 1011.

5. Kane DA, Thiagarajan RR, Wypij D, Scheurer MA, Fynn-Thompson F, Emani S, et al. Rapid-response extracorporeal membrane oxygenation to support cardiopulmonary resuscitation in children with cardiac disease. Circulation. 2010;122(11 Suppl):S241-8. doi: 10.1161/CIRCULATIONAHA.109.928390. [PubMed: 20837920].

6. Thiagarajan RR, Laussen PC, Rycus PT, Bartlett RH, Bratton SL. Extracorporeal membrane oxygenation to aid cardiopulmonary resuscitation in infants and children. Circulation. 2007;116(15):1693-700. doi: 10.1161/CIRCULATIONAHA.106.680678. [PubMed:17893278].

7. Chan T, Thiagarajan RR, Frank D, Bratton SL. Survival after extracorporeal cardiopulmonary resuscitation in infants and children with heart disease. J Thorac Cardiovasc Surg. 2008;136(4):984-92. doi: 10.1016/j.jtcvs.2008.03.007. [PubMed: 18954640].

8. Delmo Walter EM, Alexi-Meskishvili V, Huebler M, Loforte A, Stiller B, Weng Y, et al. Extracorporeal membrane oxygenation for in traoperative cardiac support in children with congenital heart disease. Interact Cardiovasc Thorac Surg. 2010;10(5):753-8. doi 10.1510/icvts.2009.220475. [PubMed: 20139198].

9. Hannan RL, Ojito JW, Ybarra MA, O’Brien MC, Rossi AF, Burke RP. Rapid cardiopulmonary support in children with heart disease: a nine-year experience. Ann Thorac Surg. 2006;82(5):1637-41. doi: 10.1016/j.athoracsur.2006.05.091. [PubMed: 17062217].

10. Chrysostomou C, Morell VO, Kuch BA, O'Malley E, Munoz R, Wearden PD. Short-and intermediate-term survival after extracorporeal membrane oxygenation in children with cardiac disease. J Thorac Cardiovasc Surg. 2013;146(2):317-25. doi: 10.1016/j.jtcvs.2012.11.014. [PubMed: 23228400].

11. Raymond TT, Cunnyngham CB, Thompson MT, Thomas JA, Dalton HJ, Nadkarni VM, et al. Outcomes among neonates, infants, and children after extracorporeal cardiopulmonary resuscitation for refractory inhospital pediatric cardiac arrest: a report from the National Registry of Cardiopulmonary Resuscitation. Pediatr Crit Care Med. 2010;11(3):362-71. doi: 10.1097/PCC.0b013e3181c0141b. [PubMed: 19924027].

12. Alsoufi B, Al-Radi OO, Nazer RI, Gruenwald C, Foreman C, Williams WG, et al. Survival outcomes after rescue extracorporeal cardiopulmonary resuscitation in pediatric patients with refractory cardiac arrest. J Thorac Cardiovasc Surg. 2007;134(4):952-959 e2. doi: 10.1016/j.jtcvs.2007.05.054. [PubMed: 17903513].

13. Paden ML, Conrad SA, Rycus PT, Thiagarajan RR, Elso Registry . Extracorporeal Life Support Organization Registry Report 2012. ASAIO J. 2013;59(3):202-10. doi: 10.1097/MAT.0b013e3182904a52. [PubMed: 23644605]

14. Gupta P, McDonald R, Chipman CW, Stroud M, Gossett JM, Imamura $\mathrm{M}$, et al. 20-year experience of prolonged extracorporeal membrane oxygenation in critically ill children with cardiac or pulmonary failure. Ann Thorac Surg. 2012;93(5):1584-90. doi: 10.1016/j.athoracsur.2012.01.008. [PubMed: 22421589].

15. Hintz SR, Benitz WE, Colby CE, Sheehan AM, Rycus P, Van Meurs KP, et al. Utilization and outcomes of neonatal cardiac extracorporeal life support: 1996-2000. Pediatr Crit Care Med. 2005;6(1):33-8. doi: 10.1097/01.PCC.0000149135.95884.65. [PubMed: 15636656].

16. Aharon AS, Drinkwater DJ, Churchwell KB, Quisling SV, Reddy VS, Taylor $\mathrm{M}$, et al. Extracorporeal membrane oxygenation in children after repair of congenital cardiac lesions. Ann Thorac Surg. 2001;72(6):2095101. [PubMed: 11789800] discussion 2101-2.

17. Duncan BW, Hraska V, Jonas RA, Wessel DL, Del Nido PJ, Laussen PC, et al. Mechanical circulatory support in children with cardiac disease. $J$ Thorac Cardiovasc Surg. 1999;117(3):529-42. [PubMed: 10047657]. 
18. Lorusso R, Gelsomino S, Vizzardi E, De Cicco G, Mastropierro R, Mariotti M, editors. Heparinless ECMO: A Feasible and effective procedure for multidisciplinary procedural complications or management. 1st Euro-ELSO Congress. 2012; .

19. Dando H, Gill M, Dittmer J. Alternative Anticoagulation for Extracorporeal Membrane Oxygenation. 7th International Conference on Pediatric Mechanical Circulatory Support Systems \& Pediatric Cardiopulmonary Perfusion. Philadelphia.

20. Mizuno T, Tatsumi E, Nishinaka T, Katagiri N, Ohikawa M, Naito H, et al. Observation of alveolar fibrosis in a goat following venoarterial bypass for up to 5 months using extracorporeal membrane oxygenation. J Artif Organs. 2004;7(2):107-9. doi: 10.1007/s10047-004-0248-x. [PubMed: 15309678].

21. Hervey-Jumper SL, Annich GM, Yancon AR, Garton HJ, Muraszko KM, Maher CO. Neurological complications of extracorporeal membrane oxygenation in children. J Neurosurg Pediatr. 2011;7(4):338-44. doi: 10.3171/2011.1.PEDS10443. [PubMed: 21456903].

22. Drews T, Stiller B, Hubler M, Weng Y, Berger F, Hetzer R. Coagulation management in pediatric mechanical circulatory support. ASAIO J. 2007;53(5):640-5. doi: 10.1097/MAT.0b013e3181492929. [PubMed: 17885339].

23. Gadepalli SK, Selewski DT, Drongowski RA, Mychaliska GB. Acute kidney injury in congenital diaphragmatic hernia requiring extracorporeal life support: an insidious problem.J Pediatr Surg. 2011;46(4):6305. doi: 10.1016/j.jpedsurg.2010.11.031. [PubMed: 21496529].

24. Smith AH, Hardison DC, Worden CR, Fleming GM, Taylor MB. Acute renal failure during extracorporeal support in the pediatric cardiac patient. ASAIO J. 2009;55(4):412-6. doi: 10.1097/MAT.0b013e31819ca3do. [PubMed: 19381083].

25. Selewski DT, Cornell TT, Blatt NB, Han YY, Mottes T, Kommareddi $\mathrm{M}$, et al. Fluid overload and fluid removal in pediatric patients on extracorporeal membrane oxygenation requiring continuous renal replacement therapy. Crit Care Med. 2012;40(9):2694-9. doi: 10.1097/CCM.ob013e318258ffo1. [PubMed: 22743776].

26. Zwiers AJ, de Wildt SN, Hop WC, Dorresteijn EM, Gischler SJ, Tibboel $\mathrm{D}$, et al. Acute kidney injury is a frequent complication in critically ill neonates receiving extracorporeal membrane oxygenation: a 14-year cohort study. Crit Care. 2013;17(4):R151. doi: 10.1186/cc12830. [PubMed 23883698].

27. Askenazi DJ, Selewski DT, Paden ML, Cooper DS, Bridges BC, Zappitelli
M, et al. Renal replacement therapy in critically ill patients receiving extracorporeal membrane oxygenation. Clin J Am Soc Nephrol. 2012;7(8):1328-36. doi: 10.2215/CJN.12731211. [PubMed: 22498496].

28. Chauhan S, Malik M, Malik V, Chauhan Y, Kiran U, Bisoi AK. Extra corporeal membrane oxygenation after pediatric cardiac surgery: a 10 year experience. Ann Card Anaesth. 2011;14(1):19-24. doi: 10.4103/09719784.74395. [PubMed: 21196670].

29. Balasubramanian SK, Tiruvoipati R, Amin M, Aabideen KK, Peek GJ, Sosnowski AW, et al. Factors influencing the outcome of paediatric cardiac surgical patients during extracorporeal circulatory support. J Cardiothorac Surg. 2007;2:4. doi: 10.1186/1749-8090-2-4. [PubMed: 17217529].

30. Sasson L, Cohen I, Tamir A, Sternfeld AR, Berlowitz Y, Lenczner O, et al. Extracorporeal membrane oxygenation in pediatric patients: our experience in the last ten years. Isr Med Assoc J. 2013;15(1):13-6. [PubMed: 23484232].

31. Black MD, Coles JG, Williams WG, Rebeyka IM, Trusler GA, Bohn D, et al. Determinants of success in pediatric cardiac patients undergoing extracorporeal membrane oxygenation. Ann Thorac Surg. 1995;60(1):1338. [PubMed: 7598575]

32. Montgomery VL, Strotman JM, Ross MP. Impact of multiple organ system dysfunction and nosocomial infections on survival of children treated with extracorporeal membrane oxygenation after heart surgery. Crit Care Med. 2000;28(2):526-31. [PubMed: 10708195].

33. Kolovos NS, Bratton SL, Moler FW, Bove EL, Ohye RG, Bartlett RH, et al. Outcome of pediatric patients treated with extracorporeal life support after cardiac surgery. Ann Thorac Surg. 2003;76(5):1435-41. [PubMed: 14602263] discussion 1441-2.

34. Delmo Walter EM, Alexi-Meskishvili V, Huebler M, Redlin M, Boettcher $\mathrm{W}$, Weng Y, et al. Rescue extracorporeal membrane oxygenation in children with refractory cardiac arrest. Interact Cardiovasc Thorac Surg. 2011;12(6):929-34. doi: 10.1510/icvts.2010.254193. [PubMed: 21429870].

35. Barsan WG, Levy RC. Experimental design for study of cardiopulmonary resuscitation in dogs. Ann Emerg Med. 1981;10(3):135-7. [PubMed: 7469152].

36. Chen YS, Chao A, Yu HY, Ko WJ, Wu IH, Chen RJ, et al. Analysis and results of prolonged resuscitation in cardiac arrest patients rescued by extracorporeal membrane oxygenation. J Am Coll Cardiol. 2003;41(2):197-203. [PubMed: 12535808]. 